\title{
Competencias emocionales y toma de decisiones responsable en preadolescentes con el apoyo de docentes, padres y madres de familia: Un estudio comparativo en estudiantes de $4^{\circ}$ a $6^{\circ}$ año de educación primaria en España
}

\author{
Emotional Competencies and Responsible Decision Making in Pre-Adolescents with the \\ Support from Teachers, Fathers, and Mothers: A Comparative Study in 4th to 6th-Grade \\ Students of Primary Education in Spain
}

Habilidades emocionais e decisões responsáveis em pré-adolescentes, com o apoio de professores, pais e mães: um estudo comparativo com estudantes de $4^{\circ} a 6^{\circ}$ ano do ensino fundamental na Espanha

Ma. Concepción Márquez-Cervantes? Universidad Complutense de Madrid Madrid, España
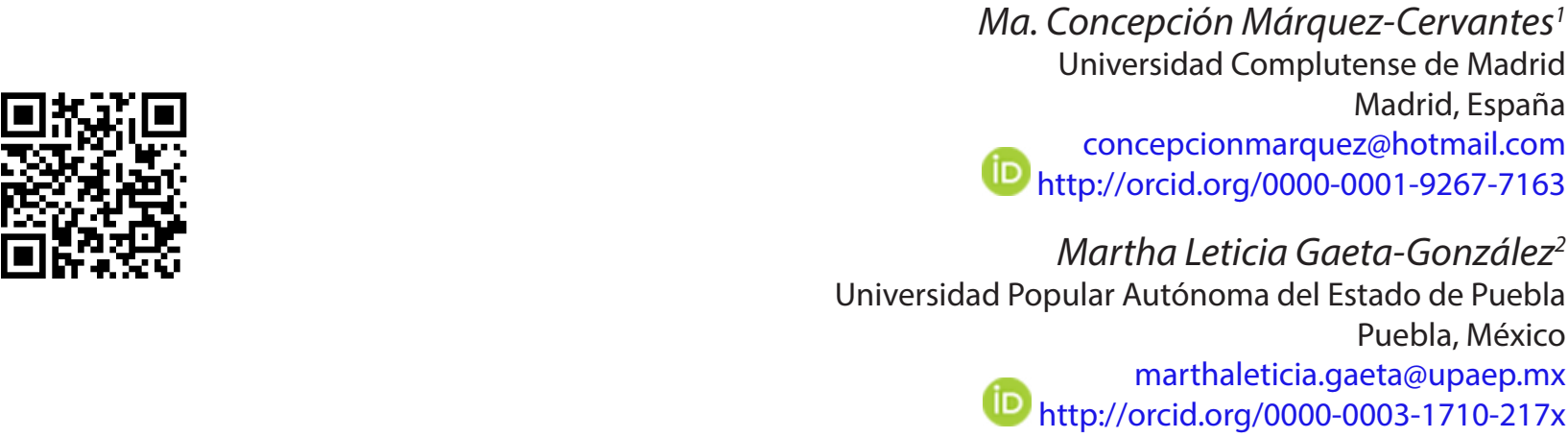

Recibido • Received • Recebido: 04 / 06 / 2016

Corregido • Revised • Revisado: 09 / 10 / 2017

Aceptado • Accepted • Aprovado: 22 / 11 / 2017

\begin{abstract}
${ }^{1}$ Estudiante de master en Inteligencia Emocional e Intervención en Emociones y Salud en la Universidad Complutense de Madrid. Maestra en Pedagogía por la Universidad Popular Autónoma del Estado de Puebla (UPAEP). Especialista en educación emocional y familia. Cuenta con publicaciones, así como con participaciones en foros nacionales e internacionales sobre este tema. Miembro del Comité Científico de la Revista A\&H Artes y Humanidades.

${ }^{2}$ Doctora en Psicología y A prendizaje por la Universidad de Zaragoza, España. Actualmente es profesora-investigadora de tiempo completo en la UPAEP. Su línea de investigación se centra en el aprendizaje autorregulado y las variables psicológicas y educativas asociadas. Ha publicado numerosas publicaciones a nivel nacional e internacional sobre el tema. Miembro de la Asociación Científica de Psicología y Educación (ACIPE) en España; miembro del Sistema Nacional de Investigadores (SNI-Nivel 1) en México; socia del Consejo Mexicano de Investigación Educativa (COMIE); miembro de la Red Mexicana de Investigadores de la Investigación Educativa (REDMIIE).
\end{abstract}


doi: http://dx.doi.org/10.15359/ree.22-1.9

URL: http://www.una.ac.cr/educare

CORREO: educare@una.cr

Resumen: Este artículo tiene como propósito analizar la relación entre las competencias emocionales y la toma de decisiones responsable en preadolescentes, y el apoyo de docentes, padres y madres de familia en este proceso, que pudiera ayudar al estudiantado a resolver problemas y enfrentarse a situaciones de riesgo de una mejor manera. Para lograrlo, se realizó una investigación con enfoque cuantitativo, de tipo descriptivo correlacional y corte transversal. Participaron 70 estudiantes de $4^{\circ}, 5^{\circ}$ y $6^{\circ}$ de primaria (entre 8 a 12 años), 3 docentes y 12 padres y madres de familia, de un colegio público en la provincia de Almería, España. Para recopilar la información se diseñaron tres cuestionarios (para el estudiantado, personal docente, padres y madres de familia), con base en modelos teóricos (Bisquerra y Pérez, 2007) e inventarios estandarizados de auto-reporte en materia de competencias emocionales (Extremera y Fernández-Berrocal, 2004; Matson, Rotatori y Helsel, 1983). Los datos colectados se analizaron mediante estadística descriptiva, de comparación entre grupos y de relación entre variables. En sus principales resultados, el alumnado reporta realizar un mayor trabajo en el fortalecimiento de su conciencia emocional, seguido por la regulación emocional y las relaciones interpersonales. No se encontraron diferencias significativas en el manejo de emociones y en la toma de decisiones responsable entre el estudiantado de los tres grados académicos; sin embargo, los varones reportaron un mayor control emocional que las mujeres. Existe una correlación significativa entre las competencias emocionales y la toma de decisiones responsable en la población preadolescentes. El personal docente manifiesta realizar un mayor trabajo en el desarrollo de la conciencia emocional del estudiantado y de apoyo en la toma responsable de decisiones. Por su parte, los padres y madres de familia buscan, en mayor medida, ayudar a sus hijos e hijas para que actúen con mayor seguridad de sí mismos y de sí mismas; además de realizar un trabajo colaborativo con la escuela, al documentarse o buscar apoyo para contribuir en la educación y desarrollo personal de su descendencia.

Palabras claves: Competencias emocionales; educación emocional; toma de decisiones; preadolescencia.

Abstract: This article aims to analyze the relationship between emotional competencies and responsible decision making in pre-adolescents, and the support of teachers and parents in this process, which could help students to solve problems and face risk situations in a better way. A quantitative, descriptive correlation and cross-sectional type research was carried out to achieve this aim. 70 students from the 4th, 5th and 6th grades (ages 8-12), three teachers and 12 parents from a public school in the province of Almeria, Spain, participated in the study. Three questionnaires were designed (for students, teachers and parents) to collect the information; they were based on theoretical models (Bisquerra \& Pérez, 2007) and standardized self-report inventories in emotional competencies (Extremera \& Fernández-Berrocal, 2004; Matson et al., 1983). The collected data were analyzed using descriptive statistics, a comparison between groups, and a correlation between variables. Among the main results, students reported that they did greater work in strengthening their emotional awareness, followed by emotional regulation and interpersonal relationships. There were no significant differences in emotional management and responsible decision making among students from the three academic years; however, males reported greater emotional control than females. A significant correlation between emotional competencies and responsible decision making in pre-adolescents was found. Teaching staff mentioned having done greater work in the development of students' emotional awareness and support of their responsible decision making. Parents, in turn, seek to help their sons and daughters to be more self-reliant, in addition to working collaboratively with the school, by documenting or seeking support to contribute to education and personal development of their children.

Keywords: Emotional competencies; emotional education; decision making; pre-adolescence. 
Resumo. O objetivo deste artigo é analisar a relação entre a competência emocional e decisões responsáveis em pré-adolescentes, e apoio de professores, pais e mães neste processo, o que poderia ajudar aos estudantes a resolver de uma melhor maneira os problemas e situações de risco. Para alcançar este objetivo, esta investigação foi levada a cabo com abordagem quantitativa, de tipo descritivo correlacional e transversal. Participaram deste trabalho 70 estudantes de $4^{\mathrm{a}}$, $5^{\mathrm{a}}$ e $6^{\mathrm{a}}$ série do ensino básico, (entre 8 a 12 anos), 3 professores e 12 pais e mães de família, de uma escola pública na província de Almeria, Espanha. Para reunir informações foram elaborados três questionários (para estudantes, professores, pais e mães), com base em modelos teóricos (Bisquerra e Perez, 2007) e inventários padronizados de auto-relato sobre competências emocionais (Extremera e FernándezBerrocal, 2004; Matson, Rotatori e Helsel, 1983). Os dados recolhidos foram analisados usando a estatística descritiva, a comparação entre grupos e a relação entre as variáveis. Em seus principais resultados, os estudantes dialogaram sobre fazer mais trabalho visando reforçar a sua consciência emocional, seguido de regulação emocional e relações interpessoais. Não houve diferenças significativas no trabalhar das emoções e tomar decisões de forma responsável entre os estudantes dos três graus acadêmicos; no entanto, os homens demonstraram maior controle emocional do que as mulheres. Existe uma correlação significativa entre as competências emocionais e decisão tomadas de forma responsável entre a população pré-adolescente. A equipe docente manifesta a necessidade de trabalhar mais no desenvolvimento da consciência emocional dos estudantes e apoiar a tomada de decisão responsável. Enquanto isso, os pais e mães de família buscam, de certa forma, ajudar seus filhos e filhas a agir com maior autoconfiança; também realizar um trabalho colaborativo com a escola, no sentido de documentação ou na busca de apoio para contribuir a educação e desenvolvimento pessoal dos seus descendentes.

Palavras-chave: habilidades emocionais; educação emocional; tomada de decisão; pré-adolescência.

\section{Introducción}

En las últimas décadas la investigación ha evidenciado que las emociones están estrechamente relacionadas con los procesos de aprendizaje que se dan en la escuela y son relevantes para una vida en plenitud (Bisquerra, 2009). De ahí que también se ha incrementado, de manera considerable, el interés por la educación de la afectividad, como un proceso educativo fundamental que se centra en la prevención y desarrollo humano (Castillo, 2013).

La educación emocional es entendida como un proceso educativo, continuo y permanente, cuyo objetivo fundamental es desarrollar las competencias emocionales que contribuyan a afrontar mejor los retos de la vida y, como consecuencia, aportar un mejor bienestar personal y social (Bisquerra y Pérez, 2007). A su vez, las competencias emocionales son consideradas competencias básicas para la vida; tal como lo plantea la Comisión Internacional sobre la Educación para el Siglo XXI, mediante el informe de la Unesco (Delors, 1996), al proponer cuatro pilares sobre los cuales debe organizarse la educación a lo largo de la vida: aprender a conocer, aprender a hacer, aprender a convivir y aprender a ser. Los dos últimos pilares representan el eje sobre el cual gira el desarrollo personal y la vida en sociedad del ser humano. 
doi: http://dx.doi.org/10.15359/ree.22-1.9

URL: http://www.una.ac.cr/educare

CORREO: educare@una.cr

Se trata, entonces, de que el proceso educativo comparta en importancia, tanto el aspecto intelectual como el desarrollo emocional, con un sentido moral (Bernal y Sandoval, 2013); con el objetivo único de que niños, niñas y jóvenes se puedan enfrentar a un mundo más complejo y competitivo, distinguiendo unas conductas de otras y con mejor oportunidad de obtener un bienestar general.

De manera específica, la preadolescencia constituye un período de profundos cambios físicos y emocionales que suelen ser difíciles para quienes los viven. Aunados a las crecientes demandas a las que el estudiantado se encuentra sometido en ambientes académicos cada vez más competitivos y a los diversos y cada vez más amplios ámbitos de decisión (Cleary y Zimmerman, 2004). De acuerdo con la Organización Mundial de la Salud (OMS), muchos de los problemas de salud mental y física de adolescentes surgen al término de la infancia y principios de la adolescencia. Esta situación puede ser potencialmente estresante y provocar inestabilidad emocional ante la incapacidad de preadolescentes para afrontar demandas y preocupaciones (Papalia, Wendkos y Duskin, 2004). En este contexto, las competencias emocionales surgen como factores protectores o preventivos ante situaciones de riesgo (Bisquerra, 2005), las cuales se pueden desarrollar tanto en el contexto escolar como en el seno familiar, de cara a una vida adulta con equilibrio y satisfacción (Cabrera, 2009).

\section{Competencias emocionales}

Diferentes investigadores han argumentado sobre una definición que aclare, de manera más precisa, el concepto de competencia emocional, el cual es aún un tema de debate entre especialistas para definirlo y delimitarlo (Bisquerra, 2009). Bisquerra y Pérez (2007) realizaron una revisión de las principales propuestas sobre competencias emocionales, a partir de las cuales desarrollan un modelo, en el que se basa el presente estudio. De manera general, las competencias emocionales se pueden definir como "el conjunto de conocimientos, capacidades, habilidades y actitudes necesarias para comprender, expresar y regular de forma apropiada los fenómenos emocionales" (Bisquerra, 2005, p. 22). En general esto implica (Bisquerra y Perez, 2007):

a) Adquirir una conciencia emocional: capacidad para reconocer los propios sentimientos y emociones, así como para percibir las emociones de las demás personas y practicar la empatía.

b) Desarrollar una regulación emocional: habilidad para manejar las emociones de manera apropiada. Darse cuenta de la interacción entre los procesos cognitivos, emocionales y de comportamiento; así como de las habilidades y estrategias que se emplean para generar emociones positivas.

c) Ampliar las competencias sociales: saber cómo relacionarse con las demás personas de manera asertiva y positiva. 
d) Adquirir una autonomía emocional: saber cómo trabajar la autogestión; es decir, el cómo se asume la responsabilidad en la toma de decisiones y la intención de implicarse en comportamientos seguros.

e) Aumentar las competencias para la vida y bienestar: habilidad para desarrollar conductas responsables, que permitan asumir el reto de organizar una vida de manera sana, equilibrada, con satisfacción y bienestar.

En este sentido, Ibarrola (2011) señala que no se nace con estas competencias, sin embargo, cada una de ellas se puede aprender, llegando a constituir un conjunto de herramientas básicas para el fortalecimiento de la eficacia personal; de no trabajar estas competencias, se fomentará lo que actualmente se conoce como "analfabetismo emocional".

Las competencias emocionales pueden enseñarse, a partir de la práctica, mediante ejemplos y vivencias (Fernández-Berrocal y Extremera, 2011). Los chicos y chicas en la preadolescencia pueden aprender a regular sus emociones observando las actuaciones de sus padres, madres y docentes, quienes se convierten en referentes principales en cuanto a actitudes y comportamientos.

Dada la función socializadora de la familia a través de la educación, el seno familiar es un contexto idóneo para el desarrollo de competencias emocionales (Bisquerra, 2012) en la infancia. Como afirma Castillo (2013, p. 164): "es en la familia, a través de los padres, como uno aprende a [desarrollarse] como persona". Por ello, una adecuada educación basada en el amor y la comprensión, a partir de una responsabilidad parental compartida, dará la seguridad y el apoyo afectivo necesarios para el crecimiento y desarrollo adecuados; para que los niños y las niñas puedan aprender progresivamente a soportar niveles de tensión, mediante el ajuste de su conducta a reglas determinadas, que les permitan favorecer la racionalidad, además de aprender a dialogar y a convivir con los demás seres (Bernal y Sandoval, 2013; Castillo, 2013).

En el contexto escolar, el papel del personal tutor/docente es fundamental para el desarrollo de competencias emocionales. Por tanto, además de poseer los conocimientos de la materia a impartir, debe ser capaz de transmitir una serie de valores al estudiantado y de trabajar sobre los procesos que faciliten la toma de decisiones con responsabilidad, ya que los chicos y las chicas pasan en las aulas gran parte de su infancia y adolescencia, periodos en los que se produce principalmente su desarrollo emocional (Extremera y Fernandez-Berrocal, 2003).

\section{Competencias emocionales y toma de decisiones responsable en la preadolescencia}

Tomar decisiones es un proceso complejo, en el que intervienen factores de índole personal e interpersonal (Bisquerra y Pérez, 2007), e implica adquirir la seguridad y la autonomía para resolver problemas y asumir las consecuencias de la actuación personal. 
doi: http://dx.doi.org/10.15359/ree.22-1.9

URL: http://www.una.ac.cr/educare

CORREO: educare@una.cr

En el contexto educativo, el proceso de toma de decisiones se trabaja generalmente a partir de la Educación Secundaria Obligatoria, principalmente a través de una orientación vocacional y profesional. Sin embargo, es clara la necesidad de contemplar en el currículo y en la actividad orientadora la capacidad de tomar decisiones, ya que esta es una tarea diaria que inicia en la infancia, se configura en la adolescencia y se define en la adultez (Álvarez y Rodríguez, 2006).

Desde este enfoque, Álvarez (2001) ha realizado estudios que han confirmado los efectos positivos del entrenamiento en el proceso de la toma de decisiones a través de la ganancia de competencias emocionales. Asimismo, el modelo afectivo-emocional de Mayer, Caruso y Salovey (1999, citado en Álvarez y Rodríguez, 2006) es uno de los más representativos en este campo, que incluye la dimensión emocional en los procesos de toma de decisiones.

Está integrado por cuatro fases: a) habilidad para reconocer las emociones y saber expresarlas; b) controlar las emociones, de tal forma que permitan un mejor proceso en la toma de decisiones; c) comprender las propias emociones y las de las demás personas; d) gestionar adecuadamente las emociones.

Tomando en cuenta que las emociones definen en gran medida las actuaciones, es importante considerar, en primer lugar, el control y la regulación de estas, si se desea realizar un proceso de toma de decisiones con responsabilidad, adecuado a cada una de las situaciones que se puedan presentar en la vida. Específicamente, en la formación de adolescentes existen varias características a considerar a fin de prepararles para tomar decisiones con responsabilidad (Álvarez y Rodríguez, 2006):

a) Promover una formación integral, diversificada e intercultural.

b) Formar en lo cognitivo, afectivo-emocional y social.

c) Fortalecer un adecuado conocimiento de sí mismo y del entorno educativo.

d) Impulsar un alto nivel de exigencia académica.

e) Ofrecer mayor libertad y responsabilidad, enfrentándolos a constantes situaciones de decisión que han de superar.

A partir de estos planteamientos se constata el papel activo de los grupos adolescentes y preadolescentes en el proceso de toma de decisiones. Desde esta perspectiva, las características del contexto escolar y del ambiente familiar tienen un papel fundamental para promover o restringir la toma de decisiones responsable. Tanto los padres y madres como los docentes, las docentes y tutores deben transformarse en guías, que transmitan la información necesaria y promuevan la reflexión en los chicos y las chicas respecto de la aceptación de sus capacidades, 
sus limitaciones y la responsabilidad que conlleva el tomar decisiones; este proceso permanente debe estar en constante revisión de cara a las eventualidades que se producen en el desarrollo del individuo (Bisquerra, 2009).

Por lo tanto, trabajar la toma de decisiones es una tarea que se lleva a cabo de forma gradual, partiendo del conocimiento de sí, del conocimiento y regulación de las propias emociones y de la empatía con las emociones de los demás sujetos, de tal forma que permita seguir conociendo las diferentes opciones que se ofrecen a lo largo de la vida.

El personal docente deberá, por tanto, promover las habilidades emocionales del estudiantado en su trabajo diario en clase (Extremera y Fernández-Berrocal, 2003). Además, es necesario que existan canales de comunicación y la acción coordinada entre el personal docente y las madres y padres de familia, a fin de que el trabajo realizado tenga continuidad (Bolívar, 2006), pues, como hemos señalado, los contextos educativo y familiar son los principales protagonistas en la educación emocional de los niños y niñas.

\section{Objetivos de investigación}

El objetivo principal de este estudio es analizar la relación entre las competencias emocionales y la toma de decisiones responsable en preadolescentes, y el apoyo del personal docente, padres y madres de familia en este proceso.

De manera específica nos propusimos:

- Conocer el nivel de desarrollo de competencias emocionales y toma responsable de decisiones del estudiantado.

- Identificar si existen diferencias en las competencias emocionales y toma responsable de decisiones entre el estudiantado de $4^{\circ}, 5^{\circ}$ y $6^{\circ}$ grado de primaria.

- Identificar si existen diferencias en las competencias emocionales y toma de decisiones responsable entre hombres y mujeres.

- Analizar la relación entre los factores de las competencias emocionales y la toma de decisiones responsable.

- Distinguir los factores queel personal docente promueveen mayor medida para el desarrollo de competencias emocionales y toma de decisiones responsable en el estudiantado.

- Distinguir los factores que los padres y madres de familia promueven en mayor medida para el desarrollo de competencias emocionales y toma de decisiones responsable en sus hijos e hijas. 
doi: http://dx.doi.org/10.15359/ree.22-1.9

URL: http://www.una.ac.cr/educare

CORREO: educare@una.cr

\section{Diseño de la investigación}

La presente investigación se ha desarrollado a partir del enfoque cuantitativo y presenta las siguientes características: es de tipo descriptivo correlacional y corte transversal. La investigación se realizó en un colegio público en la provincia de Almería, España, durante el ciclo escolar 2014-2015.

\section{Participantes}

Participaron en el estudio 70 estudiantes de $4^{\circ}, 5^{\circ}$ y $6^{\circ}$ de educación primaria (entre 8 y 12 años), 3 docentes y 12 padres y madres de familia, de un colegio público en la provincia de Almería, España. De manera específica, la muestra de estudiantes estuvo compuesta por 17 de $4^{\circ}$ grado, 28 de $5^{\circ}$ grado y 25 de $6^{\circ}$. El personal docente participante en los tres grupos estuvo conformado por 3 hombres; con edad promedio de 44.5 años, experiencia docente de más de 6 años y más de 4 años laborando en el centro educativo. Del total de padres y madres de familia, el promedio de edad de las madres fue de 37.8 años y de los padres 42.6 años. El nivel promedio de estudios de las madres era de educación secundaria obligatoria (ESO) y de los padres era de estudios en educación primaria. El método de selección de la muestra fue por disponibilidad.

\section{Instrumentos de medida}

Se diseñaron tres cuestionarios (para el estudiantado, el personal docente, los padres y las madres de familia), que consideran el modelo teórico propuesto por Bisquerra y Pérez (2007), así como dos instrumentos estandarizados sobre competencias emociones: The Matson Evaluation of Social Skills in Youngsters (MESSY) (Matson et al., 1983) y la adaptación al español del TMMS24 (Extremera y Fernández-Berrocal, 2004). A partir de estos referentes, los cuestionarios finales fueron revisados por tres personas expertas en el tema (Hambleton, 1996). En nuestro modelo teórico identificamos cinco dimensiones para el caso del alumnado: conciencia emocional, control emocional, regulación emocional, relaciones interpersonales y toma de decisiones responsable.

Matizamos que el control de las emociones implicaría desarrollar la capacidad para controlar la intensidad y duración de los propios estados emocionales, mientras que la regulación emocional correspondería a la habilidad para generar y experimentar adecuadamente las emociones y para desarrollar estrategias de afrontamiento adecuadas.

Para el caso del personal docente, madres y padres de familia se incluyeron dos dimensiones más: comunicación y disciplina; colaboración familia - escuela. A continuación, se describe la estructura final de los tres cuestionarios. 
Cuestionario para el estudiantado: dirigido a niños y niñas en etapa preadolescente (8 a 12 años). El instrumento consta de 34 preguntas integradas en cinco factores: (a) conciencia emocional (12 ítems), evalúa la percepción de las propias emociones y las de los demás; (b) control de las emociones ( 7 ítems), identifica el grado en que se modulan los propios sentimientos; (c) regulación emocional (5 ítems), identifica el grado en que se manejan las emociones de manera apropiada; (d) relaciones interpersonales (3 ítems), evalúa conductas y sentimientos en cuanto a tener amigos y amigas, compartir, etc.; (e) toma de decisiones responsable (7 ítems), evalúa el grado de seguridad y autonomía para resolver problemas y asumir las consecuencias de la actuación personal. El cuestionario se responde de acuerdo con cuatro opciones: muy rara vez (1), rara vez (2), a menudo (3) y muy a menudo (4).

Cuestionario para personal docente: dirigido a docentes de educación primaria que tienen a su cargo niños y niñas en etapa preadolescente, a fin de evaluar el trabajo realizado con el estudiantado en materia de: (a) conciencia emocional (17 ítems) evalúa el apoyo que se brinda al estudiantado para que identifique sus emociones y las de los demás individuos; (b) control emocional (7 ítems), identifica las acciones encaminadas a apoyar al estudiantado en la modulación de sus sentimientos; (c) regulación emocional (8 ítems), mide el nivel de apoyo al estudiantado para utilizar sus emociones de manera adecuada; (d) toma de decisiones con responsabilidad (14 ítems), registra las acciones encaminadas a trabajar con el estudiantado la toma de decisiones de manera autónoma y a asumir las consecuencias de sus actos; (e) comunicación y disciplina (9 ítems), identifica el grado de comunicación e involucramiento con las emociones del estudiantado y las acciones encaminadas a brindarles hábitos positivos y de orden (f) trabajo colaborativo con las familias (13 ítems), evalúa las actitudes y acciones que reflejan el trabajo en coordinación con las familias del estudiantado. Este cuestionario consta de 68 preguntas con cuatro indicadores cada una de ellas: muy rara vez (1), rara vez (2), a menudo (3) y muy a menudo (4).

Cuestionario para padres y madres de familia: dirigido a padres y madres de preadolescentes, a fin de identificar el trabajo realizado con sus hijos e hijas en materia de: (a) conciencia emocional (10 ítems), evalúa el apoyo que brindan a sus hijos e hijas para reconocer sus emociones y las de las demás personas; (b) regulación emocional ( 9 ítems), mide el apoyo que brindan a sus hijos e hijas para utilizar sus emociones de manera adecuada; (c) control emocional (9 ítems), identifica las acciones encaminadas a apoyar a sus hijos e hijas en la modulación de sus sentimientos; (d) trabajo en la toma de decisiones responsable (14 ítems), registra las acciones encaminadas a trabajar con sus hijos e hijas la autonomía en la toma de decisiones y para que asuman las consecuencias de sus actos; (e) comunicación y disciplina (11 ítems), registra el grado de interés e involucramiento emocional con sus hijas e hijos y las acciones encaminadas a establecer hábitos y rutinas positivas en sus menores; (f) trabajo colaborativo con la institución educativa de sus hijos e hijas (7 ítems), evalúa las actitudes y acciones que reflejan el involucramiento de los padres y madres en la formación académica y emocional de sus hijos e 
doi: http://dx.doi.org/10.15359/ree.22-1.9

URL: http://www.una.ac.cr/educare

CORREO: educare@una.cr

hijas. El cuestionario consta de 60 preguntas con cuatro indicadores cada una de ellas: muy rara vez (1), rara vez (2), a menudo (3) y muy a menudo (4).

\section{Recogida de la información y análisis de datos}

La aplicación del cuestionario al estudiantado se realizó en su propia aula, con una duración aproximada de 20 minutos, respetando su horario académico y estando presentes el investigador y el profesor titular. Las instrucciones fueron dadas de forma verbal al inicio de la aplicación, enfatizando la importancia de contestar con sinceridad a la totalidad de las preguntas. Se aseguró la confidencialidad de las respuestas; el estudiantado no tuvo que indicar sus datos en el cuestionario.

La administración del cuestionario al personal docente también se llevó a cabo en su propia aula, durante el horario escolar. La aplicación del cuestionario a los padres y madres de familia se realizó a través de la institución educativa, la cual solicitó que esta fuera anónima; los docentes titulares de cada grupo enviaron en sobre cerrado los cuestionarios y, de igual forma, fueron devueltos a la institución en un promedio de dos semanas.

La información fue analizada con base en el modelo teórico propuesto por Bisquerra y Pérez (2007) que plantea el desarrollo de competencias emocionales a través de la adquisición de la conciencia emocional, el desarrollo de la regulación emocional, ampliar las competencias sociales, adquirir una autonomía emocional y aumentar las competencias para la vida y bienestar; además de los postulados teóricos de Extremera y Fernández-Berrocal (2004) y Matson et al. (1983) en materia de competencias emocionales.

Los datos colectados se analizaron mediante estadística descriptiva, de comparación entre grupos y de relación entre variables. Cabe señalar que previamente se corroboró la validez de constructo y la fiabilidad de los instrumentos diseñados para este estudio. Asimismo, se realizó una exploración de la normalidad de los datos, mediante la prueba de Kolmogorov-Smirnov, la cual permite contrastar la hipótesis nula de que la muestra utilizada procede de una población con distribución normal (Pardo y Ruiz, 2005). Para el análisis de los datos se utilizó la herramienta IBM SPSS Statistics versión 20.

\section{Resultados}

\section{Análisis preliminares}

Como una primera etapa de la investigación, se corroboró la validez de constructo de los instrumentos diseñados, que reflejen los principios del marco teórico sobre el tópico a medir, mediante un análisis factorial exploratorio. Asimismo, se realizó un análisis de la consistencia interna de los tres cuestionarios, así como de cada uno de los factores que los integran, mediante el estadístico Alfa de Cronbach (ver Tabla 1).

\footnotetext{
10 Ma. Concepción Márquez-Cervantes y Martha Leticia Gaeta-González
} 
Tabla 1: Índices de fiabilidad de los cuestionarios para estudiantes, personal docente, madres y padres de familia

\begin{tabular}{|c|c|c|c|}
\hline Factor & $\begin{array}{c}\text { Estudiantes } \\
a\end{array}$ & $\begin{array}{c}\text { Personal docente } \\
a\end{array}$ & $\begin{array}{c}\text { Padres y madres de familia } \\
\qquad a\end{array}$ \\
\hline Conciencia emocional &, 67 & 84 &, 51 \\
\hline Control emocional &, 50 & 61 &, 59 \\
\hline Regulación emocional &, 54 & 77 &, 51 \\
\hline Toma responsable de decisiones &, 59 & 89 & ,60 \\
\hline Relaciones interpersonales &, 62 & No aplica & No aplica \\
\hline Comunicación y disciplina & No aplica & ,79 &, 60 \\
\hline Colaboración familia-escuela & No aplica &, 88 & 69 \\
\hline Cuestionario total & ,83 & ,96 & 82 \\
\hline
\end{tabular}

Nota: Elaboración propia.

Según la Tabla 1, en general se confirmó la estructura inicial de los tres cuestionarios y estos presentan coeficientes de fiabilidad totales aceptables (entre ,82 y ,96), aun cuando algunos factores presentan índices de fiabilidad inferiores a ,60 (Hair, Anderson, Tatham y Black, 2000). Los resultados anteriores muestran que la consistencia interna de los tres cuestionarios es suficiente y conveniente para continuar empleándolos para efectos de investigación.

\section{Resultados de estudiantes}

\section{Análisis descriptivo}

Se llevó a cabo un análisis exploratorio de los datos, respecto a las competencias emocionales y toma de decisiones responsable por parte del estudiantado, que permitiera detectar desviaciones importantes de la normalidad (outliers), además de realizar la estadística descriptiva univariante y la comprobación de los supuestos paramétricos (ver Tabla 2).

El análisis descriptivo de cada variable de estudio (ver Tabla 2) indicó que los chicos y chicas reportaron un mayor trabajo en (de mayor a menor): el factor conciencia emocional, seguido por la regulación emocional y las relaciones interpersonales, y en menor grado el control emocional y la toma responsable de decisiones. 
doi: http://dx.doi.org/10.15359/ree.22-1.9

URL: http://www.una.ac.cr/educare

CORREO: educare@una.cr

Tabla 2: Estadísticos descriptivos de los factores emocionales y toma de decisiones del alumnado $(\mathrm{n}=70)$

\begin{tabular}{lccccc}
\hline & $\begin{array}{c}\text { Conciencia } \\
\text { emocional }\end{array}$ & $\begin{array}{c}\text { Control } \\
\text { emocional }\end{array}$ & $\begin{array}{c}\text { Regulación } \\
\text { emocional }\end{array}$ & $\begin{array}{c}\text { Toma responsable } \\
\text { de decisiones }\end{array}$ & $\begin{array}{c}\text { Relaciones } \\
\text { interpersonales }\end{array}$ \\
\hline Media (M) & 37,86 & 18,93 & 15,93 & 18,67 & 10,51 \\
Mediana & 38,00 & 19,00 & 16,00 & 19,00 & 11,00 \\
Desviación típica (DT) & 4,64 & 3,26 & 2,34 & 7,79 & 3 \\
Mínimo & 12 & 7 & 5 & 28 & 12 \\
Máximo & 48 & 28 & 1,43 & 3,43 & 1,17 \\
Maritmética - M teórica & 7,86 & & & 3,01 \\
\hline
\end{tabular}

Nota: Elaboración propia.

De manera específica, en el factor conciencia emocional $(M=37,86 ; D T=4,64)$ el grupo reportó en mayor medida darse cuenta cuando un amigo o amiga se siente triste. En el factor regulación emocional $(M=15,93 ; D T=2,34)$, mencionaron que les es difícil mantener su enojo y tener días buenos muy a menudo. En el factor relaciones interpersonales $(M=10,51 ; D T=1,76)$, reportaron en mayor medida que tener amistades es muy importante. En el factor control emocional $(M=18,93 ; D T=3,26)$ indicaron, en mayor medida, que les es fácil esperar su turno. Finalmente, en el factor toma responsable de decisiones $(M=18,67 ; D T=2,79)$ mencionaron en mayor grado tomar sus propias decisiones siempre que tienen la oportunidad.

En lo que refiere al supuesto de normalidad, es decir, si la muestra procede de una población con distribución normal, se observa que para todas las variables estudiadas el estadístico de Kolmogorov-Smirnov tiene asociado un nivel crítico (significancia) menor que ,05, lo cual indica que no existe una distribución normal de los datos en dichas variables. Por ello se procedió a realizar análisis no paramétricos de los datos (Pardo y Ruiz, 2005).

\section{Comparación entre grupos}

Dado que se confirmó el no cumplimiento de los supuestos paramétricos de las variables, se realizó un análisis no paramétrico de los datos mediante la prueba Kruskal-Wallis, a fin de determinar diferencias significativas entre estudiantes según el grado académico $\left(4^{\circ}, 5^{\circ}\right.$ y $6^{\circ}$ de primaria) (ver Tabla 3). 
Tabla 3: Diferencias en los factores emocionales en el alumnado por grado académico $(n=70)$

\begin{tabular}{lcc}
\multicolumn{1}{c}{ Variable } & $\begin{array}{r}\text { Prueba de medianas } \\
\text { Conciencia emocional }\end{array}$ & $\begin{array}{c}\text { Prueba Kruskal-Wallis } \\
\text { Control emocional }\end{array}$ \\
Regulación emocional &, 923 &, 559 \\
Relaciones interpersonales &, 823 &, 773 \\
Toma responsable de decisiones &, 107 &, 608 \\
\end{tabular}

Nivel de significancia $p=, 05$

Nota: Elaboración laboración propia.

Como se observa en la Tabla 3, no se encontraron efectos significativos del grado académico sobre ninguna de las variables analizadas.

Con el propósito de evaluar las diferencias entre mujeres y varones, se efectuó la prueba estadística U de Mann-Whitney (ver Tabla 4).

Tabla 4: Diferencias en los factores emocionales en el alumnado por sexo $(n=70)$

\begin{tabular}{lcc}
\hline \multicolumn{1}{c}{ Variable } & Prueba de medianas & Prueba U de Mann-Whitney \\
& $p$ &, 923 \\
\hline Conciencia emocional &, 874 &, 017 \\
Control emocional &, 070 &, 509 \\
Regulación emocional &, 646 &, 985 \\
Relaciones interpersonales &, 883 &, 491 \\
Toma de decisiones responsable &, 874 &, 609 \\
\hline
\end{tabular}

Nivel de significancia $p=, 05$.

Nota: Elaboración propia.

Según la Tabla 4, únicamente se encontraron diferencias significativas en el control emocional $(p=, 017)$. Los varones (rango promedio $=41,77)$ reportan un mayor control emocional que las mujeres (rango promedio $=30,22$ ). 
doi: http://dx.doi.org/10.15359/ree.22-1.9

URL: http://www.una.ac.cr/educare

CORREO: educare@una.cr

\section{Análisis de correlación}

A partir de los datos anteriores, se realizó un análisis de correlación entre las variables del estudio, para el número total de sujetos que componen la muestra $(n=70)$, mediante la prueba no paramétrica Rho de Spearman (ver Tabla 5).

Tabla 5: Correlación entre las competencias emocionales y la toma de decisiones responsable $(\mathrm{n}=70)$

\begin{tabular}{|c|c|c|c|c|c|}
\hline & $\begin{array}{l}\text { Conciencia } \\
\text { emocional }\end{array}$ & $\begin{array}{c}\text { Relaciones } \\
\text { interpersonales }\end{array}$ & $\begin{array}{l}\text { Toma responsable } \\
\text { de decisiones }\end{array}$ & $\begin{array}{l}\text { Regulación } \\
\text { emocional }\end{array}$ & $\begin{array}{l}\text { Control } \\
\text { emocional }\end{array}$ \\
\hline Conciencia emocional & 1 &, $336 * *$ &, $353^{* *}$ &, $351 * *$ &, $465^{* *}$ \\
\hline $\begin{array}{l}\text { Relaciones } \\
\text { Interpersonales }\end{array}$ &, $336 * *$ & 1 &, $615^{* *}$ &, $394^{* *}$ &, $466^{* *}$ \\
\hline $\begin{array}{l}\text { Toma responsable de } \\
\text { decisiones }\end{array}$ &, $353^{* *}$ &, $615^{* *}$ & 1 &, $394^{* *}$ &, $389 * *$ \\
\hline Regulación emocional &, $351^{* *}$ &, $394^{* *}$ & $394 * *$ & 1 &, $387^{* *}$ \\
\hline Control emocional & $465^{* *}$ & $466^{* *}$ & $389^{* *}$ &, $387^{* *}$ & 1 \\
\hline
\end{tabular}

** La correlación es significativa a un nivel de 0,01 (bilateral).

Nota: Elaboración propia.

Según la Tabla 5, todas las variables que componen las competencias emocionales presentan correlación significativa positiva con la toma de decisiones responsable $(p<01)$. El mayor nivel de correlación se observa entre este último factor y las relaciones interpersonales $\left(r_{s}=, 615 ; p<01\right)$, seguido por su correlación con la regulación emocional $\left(r_{s}=, 394 ; p<01\right)$. Con una menor magnitud se observa una correlación significativa entre la toma de decisiones responsable y el control emocional $\left(r_{s}=, 389 ; p<01\right)$, seguido por la conciencia emocional $\left(r_{s}=, 353 ; p<01\right)$.

\section{Discusión de resultados}

Con base en los datos, el alumnado reporta un mayor trabajo en la conciencia emocional, seguido por la regulación emocional y las relaciones interpersonales. Estos hallazgos sugieren que el estudiantado de la muestra es consciente en cierto grado tanto de las emociones personales como de las demás personas y busca manejarlas de manera adecuada. Como indica la investigación (Fernández-Berrocal y Ramos, 2004), la conciencia en los alumnos de sus propias emociones facilita la aceptación del estado de cambios en los que se encuentran y puede apoyar en la regulación y control de sus emociones.

De manera específica, el estudiantado manifiesta el poder darse cuenta cuando un amigo o amiga se siente triste, lo que indica su sensibilidad, la empatía y consideración hacia sus 
pares, que tiene mucha relación, en el caso de este estudio, con las experiencias vividas que van acompañadas de emociones intensas y es probable que se ejerza una influencia profunda en actitudes, valores y conductas. Asimismo, los grupos preadolescentes del estudio manifestaron que el tener amistades es muy importante, lo cual corrobora que a esta edad resulta importante el reconocimiento de los demás individuos, especialmente por sus compañeras o compañeros más cercanos (Gastaminza, Vacas, Tomás y Oliva, 2007).

Por otro lado, el alumnado indica, en menor grado, controlar sus emociones y el tomar decisiones responsables. De manera específica, los preadolescentes y las preadolescentes mencionan que les es fácil esperar su turno y tomar sus propias decisiones siempre que tienen la oportunidad. Consideramos importante fortalecer estos aspectos, de tal forma que los chicos y chicas controlen sus emociones y puedan tomar decisiones sin dejarse llevar por la impulsividad que les generan sus estados emocionales, de cara a evitar situaciones de riesgo (Extremera y Fernández-Berrocal, 2004).

Al analizar las diferencias entre el estudiantado de distinto grado escolar respecto al manejo de emociones, no se encontraron diferencias significativas en ninguno de los factores. Esto indica que, independientemente de la diferencia en su edad y madurez, hay una homogeneidad en los tres grupos estudiados respecto a la conciencia emocional, el manejo de relaciones interpersonales y la toma de decisiones responsable, característicos de la etapa preadolescente, etapa evolutiva en la cual se presentan importantes cambios biológicos (Papalia et al., 2004), así como un aumento en la emotividad que se puede manifestar a través de conflictos familiares o sociales o poca adaptación a nuevos ambientes. Cabe señalar, sin embargo, que en materia de emociones no se puede evaluar en bueno o malo, sino en necesidades personales y áreas de oportunidad (Bisquerra y Pérez, 2007). Por lo que se considera prioritario que los preadolescentes y las preadolescentes desarrollen la habilidad para identificar sus emociones, a fin de poderlas regular y evitar conductas desadaptativas.

Respecto a las diferencias en el manejo de emociones entre mujeres y varones, de acuerdo con los datos encontrados, no se registran diferencias significativas en los diferentes factores, con excepción del control emocional. Se identificó un mayor control emocional en los varones, en comparación con las mujeres. Estos resultados sugieren que, aparentemente los varones demuestran tener mayor modulación en sus sentimientos y trabajar con sus procesos cognitivos. Lo anterior se encontró como una coincidencia con el desarrollo físico-psicológico de las mujeres, dado que ellas suelen iniciar cambios físicos, hormonales y psicológicos en esta etapa del desarrollo (8-9 años), un poco antes que los varones (Papalia et al., 2004), lo que repercute, quizá, en su variabilidad emocional. Lo anterior confirma la necesidad de identificar y generar conciencia de las emociones en la preadolescencia, principalmente en las mujeres, ya que estas pueden presentar sentimientos alterados, sentirse inseguras de sí mismas, etc. (Gastaminza et al., 2007). 
doi: http://dx.doi.org/10.15359/ree.22-1.9

URL: http://www.una.ac.cr/educare

CORREO: educare@una.cr

En cuanto al análisis de correlación, se puede observar que existe una correlación significativa y positiva entre todas las variables de estudio. Destaca la correlación que se observó entre el factor relaciones interpersonales y el factor toma responsable de decisiones; que refleja la importancia que tiene, para preadolescentes, el hecho de recibir reconocimiento social, la empatía que muestran hacia las personas que les rodean y la que requieren para fortalecer su autoestima, el enfoque positivo que muestran en su comportamiento y el equilibrio psicológico que reflejan en la toma de sus decisiones. En este mismo sentido, existen estudios (Extremera y Fernández-Berrocal, 2004) que han encontrado que estudiantes de primaria con puntuaciones altas en el manejo de sus emociones muestran menos agresividad con sus pares y docentes, más propensión a los comportamientos prosociales y se adaptan más para mejorar sus emociones negativas como la ansiedad o la baja autoestima.

\section{Resultados del personal docente}

\section{Análisis descriptivo}

Se llevó a cabo un análisis descriptivo de los datos, respecto a los factores que son promovidos por el personal docente para el desarrollo de competencias emocionales y toma responsable de decisiones del estudiantado (ver Tabla 6).

Tabla 6: Estadísticos descriptivos de los factores emocionales promovidos por el personal docente $(n=3)$

\begin{tabular}{|c|c|c|c|c|c|c|}
\hline & $\begin{array}{l}\text { Conciencia } \\
\text { emocional }\end{array}$ & $\begin{array}{c}\text { Control } \\
\text { emocional }\end{array}$ & $\begin{array}{l}\text { Regulación } \\
\text { emocional }\end{array}$ & $\begin{array}{c}\text { Toma responsable } \\
\text { de decisiones }\end{array}$ & $\begin{array}{l}\text { Comunicación } \\
\text { y disciplina }\end{array}$ & $\begin{array}{l}\text { Colaboración } \\
\text { con la familia }\end{array}$ \\
\hline Media (M) & 54,67 & 21,67 & 24,33 & 45,33 & 30,67 & 37,67 \\
\hline Mediana & 55,00 & 22,00 & 23,00 & 46,00 & 31,00 & 38,00 \\
\hline Desviación típica (DT) & 6,51 & 2,51 & 3,21 & 7,02 & 3,51 & 5,51 \\
\hline Mínimo & 17 & 7 & 8 & 14 & 9 & 13 \\
\hline Máximo & 68 & 28 & 32 & 56 & 36 & 52 \\
\hline$M$ aritmética - $\mathrm{M}$ teórica & 12,17 & 4,17 & 4,33 & 10,33 & 8,17 & 5,17 \\
\hline
\end{tabular}

Nota: Elaboración propia.

Según la Tabla 6, los aspectos en que el personal docente afirma trabajar en mayor medida con el estudiantado son (de mayor a menor): la conciencia emocional y la toma responsable de decisiones, seguida por la comunicación y disciplina, la colaboración con las familias, la regulación emocional y el control emocional. 
De manera específica, en el factor conciencia emocional $(M=54,67 ; D T=6,51)$, las acciones que el personal docente afirma trabajar en mayor grado son: interactuar verbalmente con sus estudiantes en forma individual durante las actividades, mostrando empatía por sus emociones y proveer explicaciones cuando se necesitan hacer cambios, atendiendo las emociones del estudiantado.

En el factor toma responsable de decisiones $(M=45,33 ; D T=7,02)$, el personal docente reporta en mayor medida: ayudar a identificar las consecuencias tanto de cumplir como de incumplir las reglas, hacer que se vivan las consecuencias y las reglas de manera constante y justa y ofrecer a los niños y niñas oportunidades para tomar decisiones.

Respecto al factor comunicación y disciplina $(M=30,67 ; D T=3,51)$, el personal docente ubicó en mayor grado: mostrar empatía y aceptación por los sentimientos de los niños y niñas, además de enseñar el cumplimiento del horario académico.

Dentro del factor colaboración con las familias $(M=37,67 ; D T=5,51)$, el personal docente reportó trabajar más los siguientes aspectos: examinar el punto de vista personal, familiar y cultural del comportamiento difícil en el estudiantado y animar a las familias a ayudar en el desarrollo del programa educativo del ciclo escolar.

En cuanto a la regulación emocional ( $M=24,33$; $D T=3,21)$, el personal docente reportó en mayor grado: apoyar a los niños y niñas cuando experimentan comportamientos difíciles y entender la relación entre el desarrollo socioemocional y el comportamiento difícil del estudiantado.

En el factor control emocional ( $M=21,67 ; \mathrm{DT}=2,51)$, el personal docente destacó: desarrollar estrategias para hacer frente a situaciones cuando los comportamientos del alumnado no son adecuados y enseñar que todas las emociones son válidas, pero no todas las expresiones son aceptables.

\section{Discusión de resultados}

Los hallazgos muestran que el personal docente considera un mayor trabajo en el desarrollo de la conciencia emocional del estudiantado, seguido por el apoyo en la toma responsable de decisiones, así como a la comunicación y disciplina. De manera específica, el personal docente busca promover la conciencia emocional en el estudiantado, principalmente mediante la interacción verbal individual con los chicos y las chicas durante las actividades; mostrando empatía por sus emociones y al proveer explicaciones cuando se necesitan hacer cambios, pues considera sus emociones. Asimismo, promueve la toma de decisiones responsable principalmente a través del establecimiento de reglas y consecuencias por el incumplimiento y al ofrecer al estudiantado oportunidades para tomar decisiones, buscando ayudarles a probar alternativas de solución. 
doi: http://dx.doi.org/10.15359/ree.22-1.9

URL: http://www.una.ac.cr/educare

CORREO: educare@una.cr

Estos resultados ponen de manifiesto el trabajo consciente del personal docente de este estudio en el reconocimiento de las emociones que sus estudiantes viven día a día en sus aulas. Con lo cual programan y establecen actividades encaminadas a trabajar las relaciones interpersonales del estudiantado y a fortalecer la autoconciencia; además de generar la toma de decisiones con responsabilidad en diversas actividades educativas. Lo anterior corrobora lo que afirman Extremera y Fernández-Berrocal (2003) en cuanto a que el personal docente constituye un agente activo de desarrollo afectivo y debe hacer un uso consciente de estas habilidades en su trabajo para la generación de un clima de seguridad, respeto y confianza entre sus estudiantes. Además, el personal docente es uno de los agentes encargados de desarrollar los procesos para la toma responsable de decisiones, de resolución de problemas y manejo de conflictos (Álvarez, 2001).

Aunado a lo anterior, aunque en menor medida, el personal docente reporta un trabajo con los padres y madres de familia de preadolescentes, principalmente, mediante el examinar el punto de vista personal, familiar y cultural del comportamiento difícil en el alumnado, animar a las familias a ayudar en el desarrollo del programa educativo del año e invitar a las familias a participar en el proceso de apoyo al comportamiento de los niños y niñas desde el inicio. En este sentido, Bolívar (2006) destaca la relevancia de la interacción conjunta entre la escuela y la familia, que promueva la puesta en común de puntos de vista y actuación, mediante una visión más amplia del proceso de formación del estudiantado. De ahí que, para que el personal docente pueda cumplir con esta función de manera eficiente, debe coordinar sus esfuerzos con los padres y madres de familia, a fin de que el trabajo realizado en clase tenga continuidad en casa.

\section{Resultados de los padres y madres de familia}

\section{Análisis descriptivo}

Se llevó a cabo un análisis descriptivo de los datos, respecto a los factores que son promovidos por los padres y madres de familia para el desarrollo de competencias emocionales y toma de decisiones responsable de sus hijos e hijas (ver Tabla 7).

Con base en el análisis descriptivo de los datos, el factor que los padres y madres de familia manifiestan promover en mayor medida en sus hijos e hijas es la conciencia emocional. Asimismo, afirman realizar trabajo colaborativo con la escuela, seguido por promover la regulación emocional y el control emocional de los niños y niñas. En menor grado reportan el trabajar la comunicación y disciplina, así como la toma responsable de decisiones. 
Tabla 7: Estadísticos descriptivos de los factores emocionales promovidos por los padres y madres de familia $(n=12)$

\begin{tabular}{lcccccc}
\hline & $\begin{array}{c}\text { Conciencia } \\
\text { emocional }\end{array}$ & $\begin{array}{c}\text { Control } \\
\text { emocional }\end{array}$ & $\begin{array}{c}\text { Regulación } \\
\text { emocional }\end{array}$ & $\begin{array}{c}\text { Toma responsable } \\
\text { de decisiones }\end{array}$ & $\begin{array}{c}\text { Comunicación } \\
\text { ydisciplina }\end{array}$ & $\begin{array}{c}\text { Colaboración } \\
\text { con la escuela }\end{array}$ \\
\hline Media (M) & 33,50 & 25,75 & 27,25 & 25,42 & 22,25 & 22,92 \\
Mediana & 34,50 & 25,50 & 27,50 & 26,00 & 22,00 & 24,00 \\
Desviación típica (DT) & 3,45 & 3,93 & 2,45 & 2,71 & 1,60 & 3,82 \\
Mínimo & 10 & 9 & 9 & 14 & 11 & 7 \\
Máximo & 40 & 36 & 36 & 56 & 44 & 28 \\
Maritmética-M teórica & 8,5 & 3,25 & 4,75 & $-9,58$ & $-5,25$ & 5,42 \\
\hline
\end{tabular}

Nota: Elaboración propia.

De manera específica, en cuanto a la conciencia emocional $(M=33,50 ; D T=3,45)$, los padres y madres de familia mencionan, en mayor medida, buscar ayudar a sus hijos e hijas para que sean más seguros de sí.

En el factor colaboración con la escuela $(M=22,92 ; D T=3,82)$, los padres y madres reportan documentarse o buscar apoyo para contribuir en la educación y desarrollo de sus hijos e hijas.

En cuanto a la regulación emocional $(M=27,25 ; D T=2,45)$, los padres y madres de familia mencionan promover en mayor medida: hablar a sus hijos e hijas en tonos tranquilos e insistir en que muestren buenos modales.

En el factor control emocional $(M=25,75 ; D T=3,93)$, los padres y madres de familia afirman trabajar en mayor grado: ayudar a sus hijos e hijas a saber cómo canalizar sus enojos y a dialogar cuando manifiestan perturbación o enojo.

En el caso del factor comunicación y disciplina $(M=22,25$; $D T=1,60)$, los padres y madres indicaron, en mayor grado, que sus hijos e hijas cuentan con hábitos de estudio y horarios para sus actividades y tomarse el tiempo para platicarles sobre acontecimientos importantes.

En el factor de toma responsable de decisiones $(M=25,42 ; D T=2,71)$, los padres y madres afirman trabajar en mayor grado el reforzar los esfuerzos de sus hijos e hijas para resolver problemas.

\section{Discusión de resultados}

Como muestran los resultados, los padres y madres de familia reportan un mayor trabajo en la conciencia emocional de sus hijos e hijas, principalmente, al buscar ayudarles para que tengan más seguridad de sí mismos o de sí mismas. Reportan, además, llevar a cabo un trabajo 
doi: http://dx.doi.org/10.15359/ree.22-1.9

URL: http://www.una.ac.cr/educare

CORREO: educare@una.cr

colaborativo con la escuela, al documentarse o buscar apoyo para contribuir en su educación y desarrollo. En menor medida, reportan trabajar en la regulación emocional de sus hijos e hijas, especialmente al procurar hablarles en tonos tranquilos e insistir en que muestren buenos modales, además de promover su control emocional, al ayudarles a saber cómo canalizar sus enojos y a dialogar cuando dicen que sienten perturbación o enojo. No obstante lo anterior, los padres y madres de familia reportan, en menor medida, trabajar la comunicación y disciplina, así como la toma responsable de decisiones.

Estos hallazgos sugieren que los padres y madres de familia son conscientes de las emociones de sus hijos e hijas y trabajan por mostrarles empatía y comprenderles. Sin embargo, requieren de un mayor trabajo encaminado a establecer el orden y a apoyar a sus hijos e hijas a que tomen decisiones con responsabilidad y a incrementar la comunicación, considerando las emociones y necesidades de los niños y niñas, dado que, de acuerdo con nuestros hallazgos, el control emocional tiene una relación significativa con el ejercicio de la disciplina y la comunicación.

Lo anterior pone de manifiesto la importancia del trabajo consciente y solidario de los padres y madres de familia (Bernal y Sandoval, 2013) a fin de que sus hijos e hijas aprendan a exponer sus puntos de vista, sus necesidades, etc. sin dejarse vencer por los sentimientos negativos. Además, el ajustarse a reglas favorece la racionalidad de los niños y niñas, al ir librándose de los propios impulsos y a aceptar que existen reglas externas que hay que cumplir (Castillo, 2013) y a generar una autonomía emocional, a partir de la instrucción directa, la observación y el modelado de sus mayores. Como afirman Bernal y Sandoval (2013, p. 145), "el proyecto familiar no supone solo un proyecto de la vida de individuos asociados afectivamente sino un plan de vida compartida de personas que trascienda a la sociedad".

\section{Visión conjunta de apoyos}

El propósito principal de este estudio fue analizar la relación entre las competencias emocionales y la toma responsable de decisiones en preadolescentes, que pudieran ayudarles a resolver problemas y a enfrentarse a situaciones de riesgo de una mejor manera, así como el papel que el apoyo del personal docente, padres y madres de familia tiene en este proceso.

A partir de los resultados, se evidencia la relación estrecha entre el conocimiento y control emocional, y la respuesta cognitiva y práctica para la toma responsable de decisiones. Al parecer, el estudiantado de la muestra es consciente, en cierto grado, de sus emociones y las de las otras personas; sin embargo, aún es necesario fortalecer el control de sus emociones y la toma responsable de decisiones. En este sentido, el desarrollo de las competencias emocionales requiere de un trabajo continuo que inicia en la infancia, se desarrolla en la adolescencia y se define en la edad adulta, por lo que, el estudiantado requiere de personal educativo emocional que, a su vez, sea un modelo de afrontamiento emocional y de habilidades interpersonales (Reina y Oliva, 2015).

\footnotetext{
20 Ma. Concepción Márquez-Cervantes y Martha Leticia Gaeta-González
}

Los artículos de la Revista Electrónica Educare del Centro de Investigación y Docencia en Educación de la Universidad Nacional, Costa Rica, se comparten bajo términos de la Licencia Creative Commons: Reconocimiento, № Comercial, Sin Obra Derivada 3.0 Costa Rica. Las autorizaciones adicionales a las aquí delimitadas se pueden obtener en el correo: educare@una.cr 
En este sentido, el personal docente reportó un trabajo en el aula para el reconocimiento de las emociones y el fortalecimiento de las relaciones interpersonales positivas entre el estudiantado, además de fomentar la toma responsable de decisiones mediante diversas actividades educativas. De esta forma, el ambiente del aula se configura como un espacio idóneo de socialización emocional, donde el personal docente con sus actitudes y comportamientos constituye el principal referente. De ahí que este último deba desarrollar, a su vez, habilidades emocionales para identificar, comprender y regular sus propias emociones, ya que estas han demostrado una incidencia en los procesos de aprendizaje, en la salud física, en la calidad de las relaciones interpersonales y en el rendimiento académico del estudiantado, así como en el desempeño laboral del personal docente (Cabello, Ruiz-Aranda y Fernández-Berrocal, 2010).

Por su parte, los hallazgos evidencian el papel de los padres y madres de familia como un referente en el desarrollo de competencias emocionales de sus hijos, principalmente al ayudarles a tener más seguridad de sí. Sin embargo, en el presente estudio, la comunicación de los padres y madres con sus hijos e hijas, así como la disciplina y la toma responsable de decisiones son aspectos que se reconoce requieren de un mayor trabajo. Por ello, se enfatiza la necesidad de que los padres y madres, como primeras personas responsables de la educación de la familia, tomen consciencia de las propias emociones, trabajen sobre ellas, y aprendan a regularlas a fin de lograr una estabilidad emocional y una vida plena. Resulta contradictorio que se desee que la descendencia tenga un buen rendimiento académico, que sea exitosa, sana y feliz, cuando como padre o madre no se tiene capacidad para generar al menos el clima propicio que ofrezca, al crecimiento de los hijos e hijas una autoestima sana, actitudes positivas que demuestren un clima afectivo, motivador y emocionalmente equilibrado.

Por otro lado, tanto padres y madres de familia como el personal docente reportan el trabajo colaborativo como un medio para el desarrollo integral de los chicos y chicas. No obstante, existen áreas de oportunidad para fomentar la cooperación entre las familias y los centros escolares, que permitan coordinar esfuerzos hacia el logro de los mismos objetivos, con una responsabilidad compartida y coordinada, hacia una educación emocional de los preadolescentes.

\section{Conclusiones}

En este trabajo hemos pretendido analizar el vínculo que guardan las competencias emocionales y la toma de decisiones responsable en preadolescentes, a partir del apoyo conjunto del personal docente, de los padres y madres de familia como personas coeducadoras, que pudiera prevenir que los chicos y chicas caigan en situaciones de riesgo y afecten su salud mental y física.

A partir de los hallazgos del estudio se confirmó que el control emocional incide en la toma responsable de decisiones, sin olvidar, por supuesto, otras competencias como la conciencia emocional y las relaciones interpersonales, competencias fundamentales en el proceso de 
doi: http://dx.doi.org/10.15359/ree.22-1.9

URL: http://www.una.ac.cr/educare

CORREO: educare@una.cr

razonamiento y en la eficiencia de los procesos de aprendizaje. Las competencias emocionales, como propósito principal de la educación emocional, tienen una gran importancia al facilitar una mejor adaptación al contexto y promover un mejor afrontamiento (Pérez-Escoda, Bisquerra, Filella y Soldevila, 2010), que se puede percibir en diversas situaciones; hemos mencionado en el presente estudio la toma de decisiones responsables. No obstante, diversos estudios (Álvarez, Bisquerra, Fita, Martínez y Pérez, 2000; Bisquerra, 2005, 2009, 2012; Extremera y FernándezBerrocal, 2003, 2004; Fernández-Berrocal y Extremera, 2011; Martínez-Otero, 2007), entre otros, han constatado sus beneficios en la resolución de conflictos, comunicación efectiva y prevención a situaciones de riesgo (uso de alcohol, uso de drogas, suicidio, violencia, etc.).

Como resultado de este estudio se pueden confirmar dos retos importantes en materia de educación emocional (Cabello et al., 2010), como son el integrar el desarrollo de competencias emocionales en el currículo formal en las diferentes etapas educativas, así como establecer programas de intervención educativa conjuntos y congruentes para padres, madres de familia y personal docente, encaminados a desarrollar competencias y habilidades emocionales en sus hijos, hijas y alumnado, de cara a la prevención de situaciones de riesgo que afecten la salud mental y física de los grupos preadolescentes.

Como hemos señalado, el desarrollo de competencias emocionales no es una labor solo del centro educativo, sino también de los padres y madres de familia; las competencias emocionales, así como las relaciones afectivas saludables, son parte de un aprendizaje que se inicia y se vive en la familia y se complementa en la escuela. De ahí la importancia de que los padres y madres de familia sean conscientes y contribuyan al desarrollo de competencias emocionales en sus descendientes y en sí mismos y mismas como parte de su formación básica y primordial en su vida.

Existen varios estudios que demuestran que las relaciones interpersonales, entre las que se cuentan las familiares, contribuyen e influyen en el estado de bienestar emocional o de felicidad de las personas (Bisquerra, 2009). Los lazos afectivos entre padres e hijos son esenciales para aprender a ser emocionalmente inteligentes. Los padres y madres deben ser conscientes, por tanto, del clima emocional que se genera en el hogar y de los efectos que pueden estar surgiendo a partir del manejo (o no) de las emociones.

Es importante señalar que, debido a la naturaleza del estudio, una muestra pequeña, en un solo colegio, los resultados no pueden ser generalizables. Por lo que es recomendable en estudios posteriores aumentar la representatividad de los sujetos participantes de manera que permita corroborar los resultados de este estudio para preadolescentes en el contexto español. Asimismo, existen limitaciones en los instrumentos que es necesario revisar, ya que no obstante, los cuestionarios completos presentan índices de fiabilidad adecuados, algunos de los factores cuentan con una fiabilidad inferior a ,60.

\footnotetext{
22 Ma. Concepción Márquez-Cervantes y Martha Leticia Gaeta-González
}

Los artículos de la Revista Electrónica Educare del Centro de Investigación y Docencia en Educación de la Universidad Nacional, Costa Rica, se comparten bajo términos de la Licencia Creative Commons: Reconocimiento, № Comercial, Sin Obra Derivada 3.0 Costa Rica. Las autorizaciones adicionales a las aquí delimitadas se pueden obtener en el correo: educare@una.cr 
No obstante lo anterior, consideramos que el presente estudio constituye un aporte para seguir fomentando el desarrollo de competencias emocionales en el alumnado dentro del contexto escolar, en coordinación con los padres y madres de familia, ya que el tomar consciencia de las propias emociones contribuye a tomar decisiones responsables a fin de aceptar y afrontar retos y vicisitudes de la vida, y para prevenir situaciones de riesgo; es pensar con el corazón en una sociedad con menos violencia, menos estrés, menos enfermedades y con mayor seguridad, satisfacciones y bienestar para las futuras generaciones.

\section{Referencias}

Álvarez, M. (Coord.) (2001). Diseño y evaluación de programas de educación emocional (2ª ed.). Barcelona: Cisspraxis.

Álvarez, M.y Rodríguez, M.L. (2006). El proceso de toma de decisiones en la educación secundaria. Un enfoque comprensivo. Revista de Orientación Educacional, 20(36), 13-38.

Álvarez, M., Bisquerra, R., Fita, E., Martínez, F. y Pérez, N. (2000). Evaluación de programas de educación emocional. Revista de Investigación Educativa, 18(2), 587-599. Recuperado de http://revistas.um.es/rie/article/view/121241/113891

Bernal, A. y Sandoval, L. (2013). "Parentalidad positiva" o ser padres y madres en la educación familiar. Estudios sobre Educación, 25, 133-149. Recuperado de https://www.unav.edu/ publicaciones/revistas/index.php/estudios-sobre-educacion/article/view/1885/1755

Bisquerra, R. (2005). Orientación y educación emocional. En J. A. Planas (Coord.), Il encuentro de orientación y atención a la diversidad (pp. 13-36). Zaragoza: Asociación Aragonesa de Psicopedagogía. Recuperado de http://www.psicoaragon.es/wp-content/ uploads/2013/07/Conclusiones Ilencuentro at div.pdf

Bisquerra, R. (2009). Psicopedagogía de las emociones. Madrid: Síntesis.

Bisquerra, R. (Coord.). (2012) ¿Cómo educar las emociones? La inteligencia emocional en la infancia y adolescencia. Esplungues de Llobregat, Barcelona: Hospital Sant Joan de Déu. Recuperado de http://faros.hsjdbcn.org/adjuntos/2232.1-Faros\%206\%20Cast.pdf

Bisquerra, R. y Pérez, N. (2007). Las competencias emocionales. Educación, 21(10), 61-82. doi: https://doi.org/10.5944/educxx1.1.10.297

Bolívar, A. (2006). Familia y escuela: Dos mundos llamados a trabajar en común. Revista de Educación, 339, 119-146. Recuperado de http://www.revistaeducacion.mec.es/re339/ re339 08.pdf 
doi: http://dx.doi.org/10.15359/ree.22-1.9

URL: http://www.una.ac.cr/educare

CORREO: educare@una.cr

Cabello,R.,Ruiz-Aranda,D.yFernández-Berrocal,P.(2010).Docentesemocionalmenteinteligentes. REIFOP, 13(1). Recuperado de http://www.redalyc.org/articulo.oa?id=217014922005

Cabrera, M. (2009). La importancia de la colaboración familia-escuela en la educación. Revista Innovación y Experiencias Educativas, 16. Recuperado de http://www.csi-csif.es/andalucia/ modules/mod ense/revista/pdf/Numero 16/MARIA CABRERA 1.pdf

Castillo, G. (2013). La normalidad afectiva en la educación de los hijos. Estudios sobre Educación, 25, 151-166. doi: https://doi.org/-DOI NO DISPONIBLE - DOI NOT AVAILABLE-

Cleary, T. J. y Zimmerman, B. J. (2004). Self-regulation empowerment program: A schoolbased program to enhance self-regulated and self-motivated cycles of student learning. Psychology in the Schools, 41(5), 537-550. doi: https://doi.org/10.1002/pits.10177

Delors, J. (Preside). (1996). La educación encierra un tesoro. Informe a la UNESCO de la Comisión Internacional sobre la Educación para el Siglo XXI. México: Ediciones Unesco.

Extremera, N. y Fernández-Berrocal, P. (2003). La inteligencia emocional en el contexto educativo: Hallazgos científicos de sus efectos en el aula. Revista de Educación, 332, 97116. Recuperado de https://www.researchgate.net/profile/Pablo Fernandez-Berrocal/ publication/39207918 La inteligencia emocional en el contexto educativo Hallazgos cientificos de sus efectos en el aula/links/587f51a308ae9275d4ede93f/Lainteligencia-emocional-en-el-contexto-educativo-Hallazgos-cientificos-de-sus-efectosen-el-aula.pdf

Extremera, N. y Fernández-Berrocal, P. (2004). El papel de la inteligencia emocional en el alumnado: Evidencias empíricas. Revista Electrónica de Investigación Educativa, 6(2), 1-17. Recuperado de http://redie.uabc.mx/redie/article/view/105/181

Fernández-Berrocal P. y Ramos N. (2004). Desarrolla tu inteligencia emocional. Barcelona: Kairós.

Fernández-Berrocal, P. y Extremera N. (2011). La inteligencia emocional como una habilidad esencial en la escuela. Revista Iberoamericana de Educación. Recuperado de http:// extension.uned.es/archivos publicos/webex actividades/4980/iegfernandez6.pdf

Gastaminza, X., Vacas, R. y Tomás, J. y Oliva, B. (2007). Aspectos psicológicos de los adolescentes. Londres: Familia Nova Schola.

Hair, J. F., Anderson, R. L., Tatham, R. L. y Black, W. C. (2000). Análisis multivariante (5ª ed.). Madrid: Prentice Hall.

Hambleton, R. K. (April, 1996). Guidelines for adapting educational and psychological tests. In NCME (Ed.), Annual Meeting of the National Council on Measurement in Education (pp. 1-39. New York, NY: NCME. Recuperado de http://files.eric.ed.gov/fulltext/ED399291.pdf 
Ibarrola, B. (Abril, 2011). Cómo educar las emociones de nuestros hijos. Conferencia presentada en el programa "Brújula en familia" en la Consejalía de Acción Social y Fundación de Ayuda contra la Drogadicción (FAD), para trabajar la prevención de las drogodependencias en el ámbito familiar. Alicante, España. Recuperado de http://www.rehueong.com.ar/ sites/default/files/ComoEducar\%20las\%20Emociones\%20de\%20NuestrosHijosB.\%20 Ibarrola 0.pdf

Martínez-Otero, V. (2007). Psicología de la inteligencia afectiva: Implicaciones pedagógicas. Revista Científica Electrónica de Psicología, 4, 246-263.

Matson, J. L., Rotatori, A. F. y Helsel, W. J. (1983). Development of a rating scale to measure social skills in children: The Matson Evaluation of social skills with youngsters (MESSY). Behavior Research Therapy, 21(4), 335-340. doi: https://doi.org/10.1016/0005-7967(83)90001-3

Reina, M. y Oliva, A. (2015). De la competencia emocional a la autoestima y satisfacción vital en adolescentes. Behavioral Psychology, 23(2), 345-359.

Papalia, D. E., Wendkos, S. y Duskin, R. (2004). Desarrollo humano. México, D.F.: McGraw-Hill.

Pardo, A. y Ruiz, M. Á. (2005). Análisis de datos con SPSS 13 base. Madrid: McGraw-Hill.

Pérez-Escoda, N., Bisquerra, R., Filella, G. R. y Soldevila, A. (2010). Construcción del cuestionario de desarrollo emocional de adultos (QDE-A). REOP, 21(2), 367-379. doi: https://doi. org/10.5944/reop.vol.21.num.2.2010.11539 